\title{
Decoding of Basic Emotions from Dynamic Visual Displays in Dementia: A Sign of Loss of Positivity Bias in Emotional Processing in Cognitively Unhealthy Aging?
}

\author{
Theofilos Gkinopoulos', Despina Moraitou ${ }^{2 *}$, Georgia Papantoniou ${ }^{3}$, \\ Magdalini Nigritinou ${ }^{2}$, Pantelis Ginos ${ }^{2}$, Daphni Kotselidou ${ }^{2}$ \\ ${ }^{1}$ School of Psychology, Panteion University, Athens, Greece \\ ${ }^{2}$ School of Psychology, Aristotle University of Thessaloniki, Thessaloniki, Greece \\ ${ }^{3}$ Department of Early Childhood Education, University of Ioannina, Ioannina, Greece \\ Email: ginopoulos@yahoo.gr, ․emorait@psy.auth.gr,gpapanto@uoi.gr
}

Received 22 June 2014; revised 22 July 2014; accepted 22 August 2014

Copyright (C) 2014 by authors and Scientific Research Publishing Inc.

This work is licensed under the Creative Commons Attribution International License (CC BY).

http://creativecommons.org/licenses/by/4.0/

(c) (i) Open Access

\begin{abstract}
Difficulties in recognizing emotional signals might have serious implications for social interactions. Neurodegenerative diseases that affect neural networks involved in emotional displays processing might thus be connected with a disproportionate impairment in social life. This study aimed at examining the ability to decode basic emotions from dynamic visual displays in mild to moderate dementia. Thirty old adults diagnosed as demented, and 30 gender-matched healthy controls were administered a measure of emotion evaluation. The groups did not differ significantly in age and educational level. The emotion evaluation test was designed to examine a person's ability to visually identify basic emotions and discriminate these from neutral expressions, when they were expressed as dynamic, subtle, day-to-day expressions. Results showed that demented participants had a great difficulty in recognizing the positively valenced emotions of happiness and pleasant surprise, while sadness, anger, and anxiety were the easiest emotions to recognize. Healthy controls were almost excellent on happiness recognition, while discrimination of non-emotional displays was the most difficult condition often mislabeled as anxiety or pleasant surprise. Results were mainly discussed in terms of socio-emotional selectivity theory positing that only older adults capable of exerting cognitive controlled favor emotional over non-emotional and positive over negative information.
\end{abstract}

\section{Keywords}

Aging, Dementia, Emotional Valence, Emotion Recognition, Socio-Emotional Selectivity Theory

*Corresponding author.

How to cite this paper: Gkinopoulos, T., Moraitou, D., Papantoniou, G., Nigritinou, M., Ginos, P. and Kotselidou, D. (2014) Decoding of Basic Emotions from Dynamic Visual Displays in Dementia: A Sign of Loss of Positivity Bias in Emotional Processing in Cognitively Unhealthy Aging? Open Journal of Medical Psychology, 3, 325-336.

http://dx.doi.org/10.4236/ojmp.2014.35034 


\section{Introduction}

Emotional signals are considered as main aspects of social communication and social functioning in general; face especially provides perceivers with a wealth of socially relevant information about another person's identity, age, sex, emotion and direction of attention. The ability to accurately recognize facial emotional expressions of basic emotions, for example happiness, surprise, fear etc., is a fundamental prerequisite for successful interaction in everyday social life. The recognition and detection of emotions from bodily expressions and different categories of postures are considered as equally important, as individuals use them to make inferences about the emotional states of others via extracting contextual information useful for an emotional interpretation. Besides, emotional postures also increase the perceived intensity of emotions and the realism of animations [1] [2]. Being able to infer what others are feeling enables us to anticipate events, avoid conflicts and regulate our emotions. Accordingly, deficits in emotion recognition can have a devastating impact on social skills, on several forms of verbal and non-verbal communication and the development and maintenance of key social relationships [3]-[6].

In corroboration of this consideration, recent research findings indicate that interpersonal difficulties and poorer quality of life in old age are associated with emotion perception difficulties. In detail, there is evidence that normal aging affects emotion recognition abilities and demonstrates substantial decline in categorization of facial expressions. Scholars in the field suggest that these declines are associated with, or stem from cognitive deficits that emerge as people grow older, from biological changes in the perceptual systems involved in detecting key facial features that communicate emotions, or from motivational shifts across the lifespan, that impact how emotional information is processed. In fact, the extant literature has confirmed that age-related deficits in fluid intelligence, speed of processing, executive functions, and working memory are related to older adults' lower performance in facial emotion recognition tasks, compared with younger ones [5] [7]. Furthermore, normal aging is considered to have an impact on frontal lobe function, which is crucial for recognizing certain negative emotions, such as anger [8] [9].

Parallel to classical cognitive deficits, several deficits can occur in specific cases, such as in early stages of Alzheimer's disease (AD) or even in Mild Cognitive Impairment (MCI) [3] [10]. Patients with AD have been shown to perform significantly worse than age-matched control participants in recognizing sad, angry, fearful, surprised, happy, and disgusted faces. However, performance can vary across the type and the modality of emotion. Moreover, Guaita et al. [11] point out that there are different and conflicting findings as regards the role of cognitive deficit and the stage of dementia in emotion recognition abilities see also [12]-[14].

Apart from these general considerations, further questions have been arisen concerning the specific categories of emotions that have been found to be difficult for detecting and recognizing. Indeed, as regards cognitively healthy older adults, compared with young adults, there is a body of literature that supports the existence of difficulties in recognizing negative emotions [8] [15] and a general preference in processing positively valenced information and recognizing more positive than negative emotions [8] [12] [16]-[21] even in older adults with amnestic MCI [13]. In fact, recent research [13] [20] [21] states the emotion of happiness as the most identified emotion among other basic emotions. Henri et al. [22] support that, on the other hand, recognition of disgust is well-preserved even in dementia cases. In general, several explanations and approaches are offered through the existed literature concerning this distinction between the capabilities to recognize positive emotions and negative emotions.

On the one hand, there are mainly some neuropsychological aspects supporting that older adults' worsening negative emotion recognition performance may stem from the decline of brain regions important for processing negative affect [23] [24]. Moreover, at this point it must be mentioned that recent research indicates that uncertainty is also a critical emotional stimulus' dimension for engaging the same brain regions (i.e., orbitofrontal cortex) that are involved in decoding - mainly but not exclusively — of negatively valenced emotional displays and that decline with age [25]-[27].

On the other hand, there is an empirically strong motivational explanation which is supported by the Socio-emotional Selectivity Theory (SST) [28] [29] according to which older adults are generally biased towards positive information processing. A large literature supports that young adults focus on emotionally negative events more than on positive ones. It has been claimed that this tendency which is designated as the "negativity bias" is over-determined and is likely adaptive [20] [30] [31]. However, on the basis of resent research, the negativity bias appears to shift with advancing age. More specifically, the respective research shows that there is a relatively higher proportion of emotional information and especially of positively valenced information com- 
pared to neutral or negatively valenced one that is remembered, recalled and recognized by older adults [20] [32]-[34]. According to the SST, this shift is due to future time perspective. When people draw near the end of their life, becoming increasingly aware of limited remaining time, and this kind of awareness leads them to prioritize emotional goals that could enhance and optimize their current experience [20] [30] [31], see also [35]. In this vein, they present a "bias" in information processing that leads them to favor emotional over non-emotional information - especially in circumstances that do not provide enough opportunities for strategic processing [32] [33], and positive over negative information [36] [37].

The last "bias" mentioned has been termed the "positivity effect" attributed to strategic processes, in sharp contrast with the assumption that the negativity bias in young adults occurs at an automatic processing level [20] [37]. Specifically, according to Kryla-Lighthall and Mather [37], there are two lines of evidence that support the assumption that older adults use cognitive control to achieve affect optimization: 1) older adults with a higher level of cognitive abilities and executive functions are more effective in emotion regulation, compared with older adults with limited cognitive resources; 2) the emergence of the "positivity effect" depends on constrains in cognition. When circumstances do not allow for goal-directed processing (i.e., splitting attention between multiple tasks), the "positivity effect" appears to be reversed to an "automatic negativity bias" in older adults' cognition [17] [37] [38]. According to Kryla-Lighthall and Mather [37], this is not surprising given that controlling affect needs increase neural activation in executive function regions including the prefrontal cortex. Considering that these regions deteriorate significantly with advancing age, older adults need to compensate for these deficits by recruiting more cognitive resources in their efforts to regulate affect, compared with young adults. Therefore, older adults are successful in affect regulation including the mechanism of the "positivity effect", only when they can devote considerable amount of cognitive control to regulation [37] [39] [40].

Furthermore, as regards the neurological functioning requirements for regulating emotions through controlled processing, there is evidence that older adults experience significant age-related losses to cognitive control structures such as the prefrontal cortex and the anterior cingulate cortex. These brain regions are considered "top-down" affect processing regions, as they are involved in higher-order cognitive processing allowing for flexible responses necessary for controlling affect [37] [39] [40]. However, there is also evidence that even if older adults experience age-related losses to cognitive control structures, healthy older adults can achieve affect optimization by recruiting additional resources to implement their regulation goals and this is clearly supported by the evidence regarding age differences in neural activation during emotion processing see for a review [37]. At this point in must be also mentioned that the primary structure that is responsible for appraising the affective quality of information rapidly and automatically ("bottom-up" processing), that is, the amygdala, remains stable with advancing age while is damaged even in the early stages of some types of dementias such as the AD [14] [39] [40].

\section{The Current Study}

In this theoretical vein, the aim of the present study was to identify the pattern of differences in basic emotion decoding from dynamic visual cues in cognitively healthy and demented older adults. Given that the emergence of the "positivity effect" became non-possible and might be reversed to an automatic "negativity bias" in older adults with limited cognitive resources, we expected demented older adults would not perform as well as cognitively healthy ones in decoding positively valenced emotional displays and especially positive displays that were not characterized by uncertainty (Hypothesis 1). We also expected demented older adults to have higher performance in decoding negatively valenced emotional displays, compared to healthy older adults (Hypothesis 2). Moreover, given that affect optimization was also associated with favoring emotional over non-emotional information processing, a third hypothesis was formulated. According to Hypothesis 3, cognitively healthy older adults would not perform as well as demented ones on recognizing emotionally neutral displays, due to the tendency to detect emotion in non-emotional displays.

\section{Materials and Methods}

\subsection{Participants and Procedure}

As regards the sample of the study, there were 30 participants diagnosed as demented (11 men, 19 women) and 30 gender-matched healthy controls. All participants were aged 67 and over. The participants of the demented 
group were staying within the places of hospitality mentioned below. The two groups did not differ significantly in age, $t(58)=1.57, p>0.05$. Demented group: $M=78$ years, $S D: 4.86$, Age-range: 68 - 86 years; Healthy controls: $M=76.03$ years, $S D$ : 4.84, Age-range: $67-85$ years. They also did not differ significantly in educational level (EL), $t(58)=-0.39, p>0.05$. EL was defined according to the number of years of education. Demented group: EL 1 ( 0 - 6 years) and $n=15$, EL 2 ( $7-12$ years) and $n=14$, EL 3 ( $\geq 13$ years) and $n=1$; Healthy controls: EL 1 ( 0 - 6 years) and $n=16$, EL $2(7-12$ years) and $n=10$, EL 3 ( $\geq 13$ years) and $n=4$.

The demented group was recruited from two places of hospitality for elderly people. The first place was a boarding house of the parish church of St. Paraskevi in Athens. The second place was a center of elderly people's hospitality in Halkis (Euboea). The demented participants were diagnosed by consultant neurologists and psychiatrists and met diagnostic criteria for possible dementia as established by both neurological assessment using Magnetic Resonance Imaging and cognitive assessment via the Mini Mental State Examination (MMSE) tool. They had MMSE scores between 13 and 20, falling in the mild to moderately demented range.

The healthy control participants were community dwelling adults - volunteers recruited by the researchers through seniors' centers. Potential participants in the healthy control group were excluded if they had MMSE scores of less than 27.

Exclusion criteria for all groups were history of neurological conditions or psychiatric diseases, alcohol or drug abuse, severe head trauma, profound visual impairments, verbal incomprehension and a Geriatric Depression Scale (GDS) - 15 score of over than 6.

Participants were examined at an individual basis either at the center recruited or in their own home. For all control and demented participants informed consent was obtained and then they completed an individual-demographics form. All the participants additionally completed the Greek version of the Mini Mental State Examination MMSE [41] [42]. The MMSE was used to provide an estimate of overall cognitive functioning. They also completed the Greek version of the Geriatric Depression Scale-15 (GDS-15 [43] [44]) to assess current depression symptoms. This was followed by an assessment of emotion recognition as measured by the Emotion Evaluation Task of the TASIT [15] [45]-[47].

\subsection{Instrument: The Emotion Evaluation Task (EET-FORMA) [15] [45]-[47]}

To examine the ability to decode basic emotions from ecologically valid cues, the "TASIT PART I: Emotion Evaluation Task (EET-FORM A)" was used. The EET is the first part of a broader audiovisual tool designed for the clinical assessment of social perception that is called "The Awareness of Social Inference Test (TASIT)". The EET was designed to examine a person's ability to identify six basic emotions, namely happiness, pleasant surprise, sadness, anger, anxiety, and disgust, and discriminate these from neutral expressions, when they are portrayed dynamically by professional actors. Specifically, it comprises 28 alternative forms of a series of short (15 - 60 seconds) videotaped vignettes of people interacting in everyday situations. In fact, "people" are actors trained in the "Method" style that requires the actor to elicit a real emotion in himself/herself. At this point it must be mentioned that actors were specifically trained not to provide the viewer with unlimited exposure to an emotional expression, because, in real life, displays of genuine emotion are fleeting and evolving and usually mediated by a tendency to "play against" expressions of strong emotion. Besides actor's style, the EET uses visual and auditory contextual cues to assess emotion decoding systematically.

In some scenes there is only one actor talking. Other scenes depict two actors. In these cases instructions are given to focus on one of them. The "target" actor in each scene enacts the script according to one of the six basic emotions or no particular emotion (the "neutral" condition). After viewing each scene the participant is asked to choose the emotion displayed by the actor from a list of six emotional categories and one non-emotional category (neutral) displayed in random order on one of five Response Cards.

The 28 scenes comprise four portrayals of each emotion including the "neutral" condition, in quasi-randomized order. At this point it must be mentioned that all scripts are neutral in content and do not lend themselves to any specific emotion. However, considering that the test was developed in English, for the purposes of the present study, we decided to administer it with the sound turned off, so as to focus on the person's ability to read dynamic visual cues. Administration of EET typically took between $15-25$ min, depending on the participant's age. The EET was administered according to the standard procedure outlined in the relevant manual. A practice item preceded the main task to familiarize the participant with the task requirements.

As regards the psychometric qualities of the EET, based on the performance of adults with severe traumatic 
brain injury, a reliability estimate for test-retest was found in the moderate level $(r=0.74)$. In relation to EET's construct validity, it was found that it was significantly correlated with premorbid IQ, information processing speed, new learning of socially relevant information and specifically faces, executive function as measured by a socially relevant task and a geometric problem solving task measuring visuospatial and analogical reasoning, and the Ekman photo labeling and matching tasks as measures of social perception [15] [45]-[47].

\section{Results}

EET data were firstly analyzed with a $2 \times 7$ mixed ANOVA with the between-subjects variable of group status (demented-healthy older adults) and the within-subjects variable of emotion type (happy, surprised, angry, anxious, sad, revolted, and neutral). These analyses indicated that there was a main effect of group status, $F(1,58)$ $=7.69, p=0.007, \eta_{p}^{2}=0.12$, and of emotion type, $F(6,348)=11.71, p<0.001, \eta_{p}^{2}=0.17$. There also was a large interaction effect, $F(6,348)=26.26, p<0.001, \quad \eta_{p}^{2}=0.31$.

To analyze the interaction between group and emotion type observed for EET, tests of simple effects were conducted. For four of the emotions and the neutral condition, group was a significant simple main effect in the alpha level of 0.001: Happiness, $F(1,58)=90.85, p<0.001$; Anger, $F(1,58)=18.72, p<0.001$; Anxiety, $F(1$, $58)=23.42, p<0.001$; Sadness, $F(1,58)=28.48, p<0.001$; Neutral condition, $F(1,58)=11.46, p=0.001$. Relative to healthy controls, the demented group had a significantly lower ability of correct recognition of happiness but a significantly higher ability to recognize the three negatively valenced emotions of anger, anxiety and sadness as well as to discriminate the non-emotional or neutral visual displays. In addition, the findings showed that there was a tendency for better recognition of disgust in the demented group compared to healthy controls: Disgust, $F(1,58)=5.66, p=0.02$. The exception was pleasant surprise, where group was clearly not a significant simple main effect, $F(1,58)=0.91, p=0.345$.

Further tests of simple effects revealed that emotion type was a significant simple main effect within each group: Demented participants, $F(6,174)=25.77, p<0.001, \eta_{p}^{2}=0.47$; Healthy elderly subsample, $F(6,174)$ $=13.35, p<0.001, \eta_{p}^{2}=0.32$. In terms of the pattern of these simple effects (see Figure 1), demented participants had a great difficulty recognizing happiness, $M=1.30, S D=1.05$. The other one positively valenced emotion, that is pleasant surprise, was the second most difficult emotion to recognize, $M=1.70, S D=1.26$. On the other hand, sadness, $M=3.63, S D=0.76$, anger, $M=3.17, S D=0.70$, and anxiety, $M=3.07, S D=0.87$, were the first, second, and third easiest emotions to recognize, respectively, with disgust, $M=2.93, S D=0.91$, and the neutral condition, $M=2.30, S D=1.02$, to follow them.

Inversely, healthy controls were almost excellent in happiness recognition, $M=3.53, S D=0.73$, while discrimination of non-emotional displays (neutral condition) was by far the most difficult condition for them, $M=$ $1.33, S D=1.18$. Sadness, $M=2.33, S D=1.09$, disgust, $M=2.23, S D=1.33$, anger, $M=2.10, S D=1.16$, pleasant surprise, $M=2.00, S D=1.17$, and anxiety, $M=1.90, S D=0.99$, in descending order of recognition, fell between them (see Figure 2).

On the basis of the aforementioned findings, it was considered useful to assess whether any of the group differences in the worst recognized emotion by the demented group, that is happiness, and the worst recognized

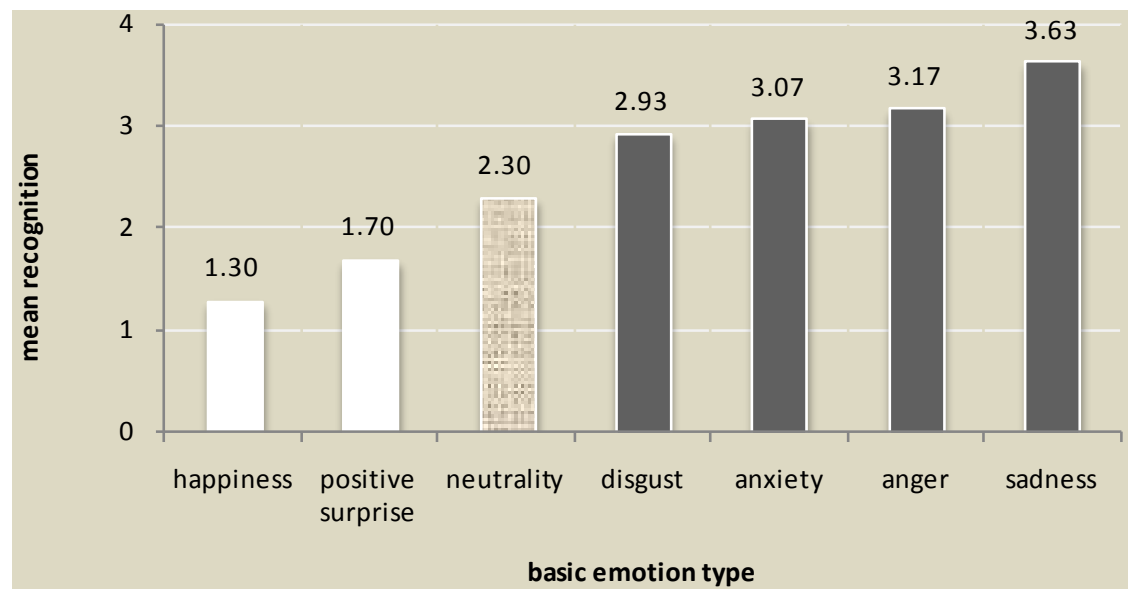

Figure 1. Decoding of basic emotions from dynamic visual cues in dementia. 


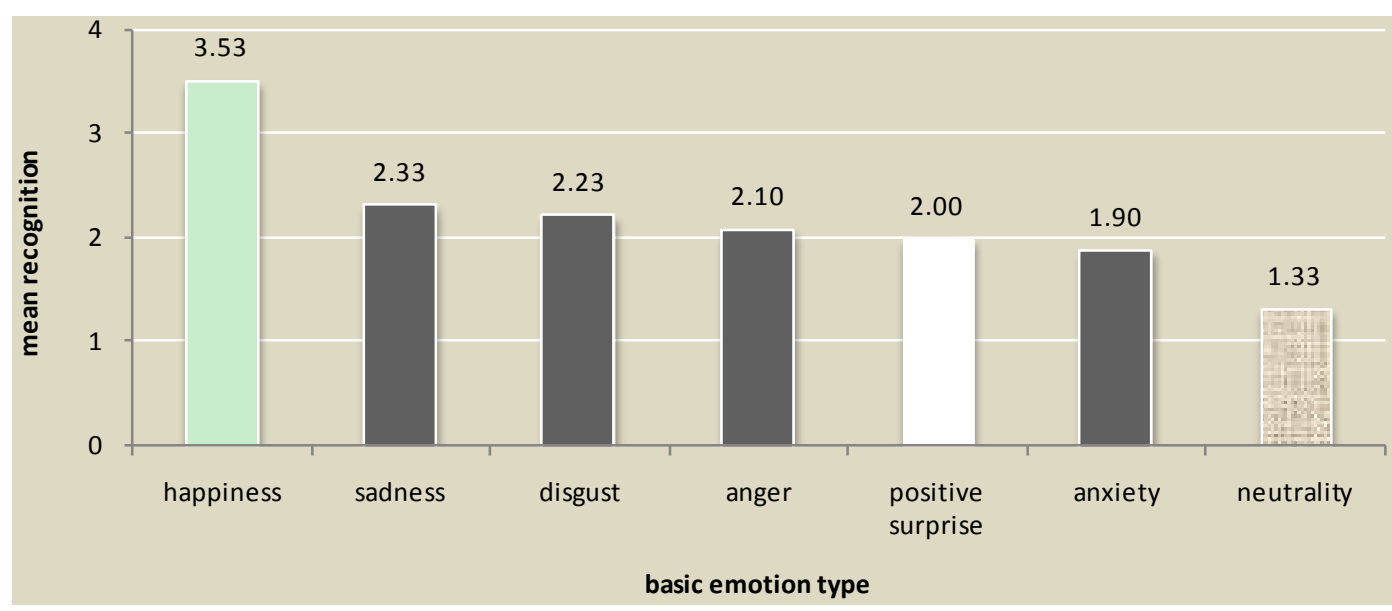

Figure 2. Decoding of basic emotions from dynamic visual cues in cognitively healthy aging.

condition by the cognitively healthy group, that is non-emotional displays, reflected differences in patterns of error responses. Inspection of the percentage of error types in relation to the four EET scenes with portrayals of happiness indicated that the most commonly made error in the demented group was to label happiness displays as pleasant surprise ones (see Table 1). Inspection of the percentage of error types in relation to the scenes including emotionally neutral portrayals indicated that a systematic error in the demented group was to label emotionally neutral displays as displays of sadness, whilst the cognitively healthy group is presented with a more heterogeneous pattern of error responding. In fact, healthy older adults most often mislabeled neutrality as anxiety or pleasant surprise (see Table 1).

Subsequently, Pearson correlations were computed to examine whether total error responses as regards happiness and non-emotional displays recognition, were correlated with correct decoding of each of the six target emotions and the neutral condition. Whilst significant associations were observed in the demented group between recognition of happiness and error responses $(1=$ correct response; $2=$ wrong response $)$ for each one of the four scenes with portrayals of happiness, $r=-0.79, p<0.001, r=-0.56, p=0.001, r=-0.56, p=0.001, r=$ $-0.55, p=0.002$, respectively, the fact that the correlations were negative simply indicates that error responses were associated with fewer correct identifications of happiness. Taking into account the findings presented in Table 1 for the demented group, the significant correlation found between recognition of pleasant surprise and error responses for the third scene with happiness portrayals, $r=-0.61, p<0.001$, indicates that misuse of the label "surprised" was associated with fewer correct identifications of surprise overall. In other words, a probably preferential use of the 'surprised' label was not associated with correct identification of surprise in the demented group. Finally, the only positive correlation found was between recognition of anger and error responses for the first scene with happiness portrayals in the demented group, $r=0.47, p=0.008$. This finding may be indicative of an overall prevalence of negativity bias in cognitively unhealthy elderly that makes it difficult to identify happiness.

\section{Discussion}

The present study aimed at identifying the pattern of differences in basic emotions' decoding from dynamic visual cues in cognitively healthy and demented older adults. The first hypothesis formulated was that demented older adults would not recognize positively valenced emotional displays as well as cognitively healthy ones (Hypothesis 1). Inversely, it was hypothesized that they would perform higher than cognitively healthy older adults in the recognition of negatively valenced emotional cues (Hypothesis 2). Indeed, the results showed that patients with dementia were significantly impaired in happiness decoding from dynamic visual cues-systematically tending to confuse happiness with pleasant surprise - compared to cognitively healthy older adults. These, on the other hand, had an almost excellent performance on happiness recognition. Moreover, pleasant surprise, the second positively valenced emotion examined in this study, was also the second most difficult emotion to recognize in the case of the demented group. Inversely, as expected, relatively to healthy controls, the demented group was found to have a significantly higher ability to recognize the four negatively valenced emotions 
Table 1. Correct and erroneous identifications of dynamic happiness displays and non-emotional (neutral) cues in demented and cognitively healthy elderly.

\begin{tabular}{|c|c|c|c|c|c|c|c|}
\hline \multirow{2}{*}{$\begin{array}{l}\text { Target emotion } \\
\text { Scenes } 1 \text { - } 4\end{array}$} & \multicolumn{7}{|c|}{ Emotion label selected (percentage) } \\
\hline & Happiness & Positive surprise & Neutral & Sadness & Anger & Anxiety & Disgust \\
\hline \multicolumn{8}{|l|}{ Happiness 1} \\
\hline Demented & $30 \%$ & $46.7 \%$ & $23.3 \%$ & & & & \\
\hline Healthy & $73.3 \%$ & $13.3 \%$ & & $3.3 \%$ & $6.7 \%$ & $3.3 \%$ & \\
\hline \multicolumn{8}{|l|}{ Happiness 2} \\
\hline Demented & $16.7 \%$ & $66.7 \%$ & $16.7 \%$ & & & & \\
\hline Healthy & $90 \%$ & $10 \%$ & & & & & \\
\hline \multicolumn{8}{|l|}{ Happiness 3} \\
\hline Demented & $16.7 \%$ & $73.3 \%$ & $6.7 \%$ & & & & \\
\hline Healthy & $93.3 \%$ & $6.7 \%$ & & & & & \\
\hline \multicolumn{8}{|l|}{ Happiness 4} \\
\hline Demented & $66.7 \%$ & $23.3 \%$ & $10.0 \%$ & & & & \\
\hline Healthy & $93.3 \%$ & $6.7 \%$ & & & & & \\
\hline \multicolumn{8}{|l|}{ Neutral 1} \\
\hline Demented & & $10 \%$ & $56.7 \%$ & $23.3 \%$ & & $10 \%$ & \\
\hline Healthy & & $16.7 \%$ & $26.7 \%$ & $16.7 \%$ & & $40 \%$ & \\
\hline \multicolumn{8}{|l|}{ Neutral 2} \\
\hline Demented & & & $63.3 \%$ & $30 \%$ & & $6.7 \%$ & \\
\hline Healthy & $10 \%$ & $33.3 \%$ & $36.7 \%$ & $10 \%$ & $3.3 \%$ & $6.7 \%$ & \\
\hline \multicolumn{8}{|l|}{ Neutral 3} \\
\hline Demented & & $10 \%$ & $46.7 \%$ & $43.3 \%$ & & & \\
\hline Healthy & & $10 \%$ & $36.7 \%$ & $16.7 \%$ & $3.3 \%$ & $23.3 \%$ & $10 \%$ \\
\hline \multicolumn{8}{|l|}{ Neutral 4} \\
\hline Demented & & & $60 \%$ & $26.7 \%$ & & $13.3 \%$ & \\
\hline Healthy & & $26.7 \%$ & $33.3 \%$ & $13.3 \%$ & $6.7 \%$ & $20 \%$ & \\
\hline
\end{tabular}

examined in this study, and mainly anger, anxiety, and sadness. Hence, the current findings seem to confirm the theoretical account of older adults' positivity bias, known as "the cognitive control model" [37] [48].

According to the broader line of reasoning of the Socio-emotional Selectivity Theory, older adults' positivity effect is due to their greater focus on regulating emotions compared to young adults, in order to enhance their current experience, because they are aware of their limited remaining time in life [19] [28] [29] [34] [35]. The cognitive control model claims that this kind of demanding self-regulation relies on brain-resource-dependent cognitive control processes that take place in healthy aging [37] [48]. Specifically, recent research shows that healthy older adults, compared to young adults, show increased prefrontal cortex (PFC) or anterior cingulate cortex (ACC) activity together with decreased amygdala activity while processing negative stimuli. Hence, age-related decreases in amygdala activity in healthy aging are not due to inherent amygdala impairments but to strategic processes based on PFC or ACC recruitment [48]. On the other hand, research on Alzheimer's disease shows that disease-related amygdala decline leads to hyperactive amygdala responses to neutral and fearful human faces [48]. In the same vein, research examining how strategic abilities and goal structure may interact to 
affect success at emotion regulation, shows that, although the emotion-focused goals of older adults may be chronically and automatically activated —operating as a default at an unconscious level, when cognitive control resources are not available to guide behavior in a goal-consistent manner, emotional goals may have unintended consequences. In such a context, no control processes may be available to suppress goal-inconsistent information, one it has been detected. In this vein, the "positivity effect" related to emotional goals of older adults can be reversed to an over-determined "negativity bias" [17] [37] [49]. Based on the findings of this study, it seems that cognitively healthy older adults display a relatively clear "positivity effect" in the case of basic emotions decoding from dynamic visual cues, which is reflected in their excellent ability to recognize happiness together with their lower ability to recognize the negatively valenced emotions, compared to the demented older adults. Inversely, the results of this study suggest that demented persons, due to their diminished cognitive control resources, appear to reverse the "positivity effect" to an automatic "negativity bias" in basic emotions decoding from dynamic visual cues. This is mainly reflected in their significantly lower ability to recognize happiness and their higher ability to decode negatively valenced emotions, compared to healthy older adults.

As regards the specific type of basic emotion, it is worth noting that, according to the findings of this study, happiness recognition is not preserved even in mild dementia. Given that the same ability is very-well-preserved in cognitively healthy aging, happiness decoding from dynamic visual cues might be examined as a marker of relatively early cognitive decline, since it could reflect the loss of positivity bias in cognitively unhealthy aging.

Besides happiness, the "picture" of findings regarding the second positively valenced emotion examined in this study, that is pleasant surprise, seems to be more complicated. On the one hand, the "positivity effect" displayed by cognitively healthy older adults appears not to be so "beneficial", in order for them to systematically recognize pleasant surprise. On the other hand, the "negativity bias" displayed by demented persons seems to be somewhat "detrimental", in order for them to recognize pleasant surprise. Finally, based on overall performance of each group, pleasant surprise decoding from dynamic visual displays appears problematic in both cases. Moreover, given the high rate of the confusion of happiness with pleasant surprise in the case of the demented older adults as well as the high rate of the confusion of non-emotional displays with displays of pleasant surprise in healthy older adults, it is obvious that pleasant surprise, at least as it is displayed dynamically in the EET, is rather considered a mixed emotion. As Moraitou et al. [15] mention, unpredictability or unexpectedness is inherent in the "nature" of surprise. Thus, even if surprise is pleasant, it seems that inherent uncertainty interacts with the positive valence of the emotion and this seems to seriously affect recognition and identification of pleasant surprise.

Besides specific emotions and specific biases related to emotional valence, the Socio-emotional Selectivity Theory posits that older adults' shift in goals is associated with broader systematic biases in cognition that favor emotional over non-emotional information, in general [17] [20] [28]. On the same wavelength, a series of empirical studies indicated that, when emotional stimuli are relatively low-arousing, older adults display a broader emotion effect compared to younger adults, but with no evidence of emotional preference see [13] [20]. In this light, in confirmation of Hypothesis 3, it seems that the high level of difficulty of cognitively healthy older adults in discriminating non-emotional displays and their tendency to confuse emotionally neutral cues mainly with anxiety and pleasant surprise could be interpreted as a general bias which consists in perceiving emotion at least in non-emotional displays that are ambiguous enough. Inversely, both the higher performance of demented persons in discriminating non-emotional portrayals and their tendency to confuse neutrality with sadness, as well as the positive relationship between their ability to recognize anger and error responses for one of the scenes with happiness portrayals, could be indicative of the unavailability of cognitive control processes that can suppress motivation-inconsistent information in persons diagnosed with mild to moderate dementia.

At this point it should be pointed out that there seems to be an inconsistency between the findings of this study and some previous findings on emotion recognition in dementia [11] [12] [22] [50]. This may be partially explained by the type of the task used in this study to measure emotion recognition ability. The vast majority of neuroimaging and neuropsychological studies of emotion recognition have focused on the ability to decode an emotion shown in static photographs of facial expressions. However, successful navigation of the social world requires decoding multiple modes of emotional information, as this information unfolds dynamically. According to previous studies which investigated the neural regions involved when people decode emotions that unfold and change dynamically, neither amygdala nor temporal lobe atrophy was associated with impaired performance on emotion recognition. However, frontal lobe regions such as the orbitofrontal cortex and the gyrus rectus were found to be involved in dynamic emotion recognition [51]. At a broader level, strategic emotion regulation associated with motivational shifts in older age has been empirically associated with dorsomedial, dorsolateral, and 
ventrolateral prefrontal cortex and posterior parietal cortex, that is, with areas that support cognitive control processes in general [52]. Moreover, at the behavioral level, it has been found that as intensity of an emotion to be identified is reduced, the difficulty of both cognitively healthy and demented older adults in emotion recognition is increased [2] [22]. The EET is designed to examine a person's ability to decode basic emotions from dynamic displays of genuine emotions that are fleeting and evolving [46] [47]. Hence, the differences between the findings of this study and previous ones may be partially explained by the different brain regions that are recruited to decode dynamic and subtle emotions, compared to those activated to recognize static and intense emotions. Indeed, there is at least one previous study in which it was found that individuals with Alzheimer's disease did not differ from age-matched controls on the EET while they significantly differ from them on a test of emotion decoding from still images [22], see also [53].

\section{Limitations and Future Directions}

Our research findings must be interpreted with several limitations in mind: 1) our sub-sample of demented participants including a diverse set of dementia types and the diagnosis was mainly based on neurological and psychiatric examinations. Accordingly, this approach lacks power to reveal the pattern of differences in emotion decoding from dynamic visual displays between persons diagnosed with a specific type of dementia and healthy controls, or among older persons diagnosed with different types of dementia; 2) the restricted nature of the sample should be also noted with regard to the number of participants in each group; 3 ) an additional limitation is the cross-sectional design of the study; it is unknown if the same pattern of results would be obtained if the same persons were repeatedly measured; for example, longitudinal follow-up of performance during the course of a specific type of dementia would improve understanding of emotion recognition in cognitively unhealthy older population; 4) finally, demented participants were all staying within the places of hospitality. Due to this stay, they may experience more negative emotions than healthy individuals do. So, they may also identify more easily negative than positive emotions, compared to healthy individuals. Results from this study would be also strengthened by exploring systematically the cognitive and perceptual components of emotion recognition in cognitively healthy and unhealthy older adults, through the use of a comprehensive neuropsychological battery, through manipulations in order to change the cognitive or perceptual load of emotion recognition tasks as well as by examining multiple varieties of emotion tracking. Generally speaking, more rigorous methods are needed to examine the effects of normal and pathological aging on emotion recognition as well as to depict in more details the specific impacts and influences of environmental factors on the emotion recognition and decoding abilities of individuals.

\section{Conclusion}

The present findings provide important information about the pattern of differences in dynamic emotion recognition in cognitively healthy and demented older adults. The major conclusion of the study is that cognitively healthy older adults, due to motivational shifts related to future time perspective that is perceived as limited, display a preference in processing positively valenced information, which is known as the "positivity effect" bias, with regard to basic emotions decoding from dynamic visual cues. This bias is mainly reflected in their excellent ability to recognize happiness together with their lower ability to recognize basic negatively valenced emotions, compared to demented older adults. Inversely, demented persons, probably due to their diminished cognitive control resources, appear to reverse the "positivity effect" to an over-determined "negativity bias" in basic emotions' decoding from dynamic visual cues. This is primarily reflected in their significantly lower ability to recognize happiness and their higher ability to decode three negatively valenced emotions, namely, anger, sadness, and anxiety, compared to cognitively healthy older adults.

\section{Acknowledgements}

This work was supported by the Research Committee of Aristotle University of Thessaloniki, Greece (Special Account for Research Implementation/87871/Moraitou).

\section{Disclosure}

The authors declare that there is no conflict of interests regarding the publication of this paper. 


\section{References}

[1] Buisine, S., Courgeon, M., Charles, A., Clavel, C., Martin, J.C., Tan, N. and Grynszpan, O. (2014) The Role of Body Postures in the Recognition of Emotion in Contextually-Rich Scenarios. International Journal of Human-Computer Interaction, 30, 52-62. http://dx.doi.org/10.1080/10447318.2013.802200

[2] Dael, N., Goudbeek, M. and Scherer, K.R. (2013) Perceived Gesture Dynamics in Nonverbal Expressions of Emotion. Perception, 42, 642-657. http://dx.doi.org/10.1068/p7364

[3] McCade, D., Savage, G., Guastella, A., Hickie, B.I., Lewis, J.G.S. and Nailsmith, L.S. (2013) Emotion Recognition in Mild Cognitive Impairment: Relationship to Psychosocial Disability and Caregiver Burden. Journal of Geriatric Psychiatry and Neurology, 26, 165-173. http://dx.doi.org/10.1177/0891988713491832

[4] Monroe, F.J., Griffin, M., Pinkham, A., Loughead, J., Gur, R.C., Roberts, P.L.T. and Edgar, J.C. (2013) The Fusiform Response to Faces: Explicit Versus Implicit Processing of Emotion. Human Brain Mapping, 34, 1-11. http://dx.doi.org/10.1002/hbm.21406

[5] Suzuki, A. and Akiyama, H. (2013) Cognitive Aging Explains Age-Related Differences in Face-Based Recognition of Basic Emotions Except for Anger and Disgust. Aging, Neuropsychology and Cognition, 20, 253-270. http://dx.doi.org/10.1080/13825585.2012.692761

[6] Slessor, G., Riby, M.D. and Finnerty, N.A. (2013) Age-Related Differences in Processing Face Configuration: The Importance of the Eye Region. The Journals of Gerontology Series B: Psychological Sciences and Social Sciences, 68 , 228-231. http://dx.doi.org/10.1093/geronb/gbs059

[7] Mienaltowski, A., Johnson, R.E., Wittman, R., Wilson, A.T., Sturycz, C. and Farley Norman, J. (2013) The Visual Discrimination of Negative Facial Expressions by Younger and Older Adults. Vision Research, 81, 12-17. http://dx.doi.org/10.1016/j.visres.2013.01.006

[8] Krendl, A.C. and Ambady, N. (2010) Older Adults' Decoding of Emotions: Role of Dynamic Versus Static Cues and Age-Related Cognitive Decline. Psychology and Aging, 25, 788-793. http://dx.doi.org/10.1037/a0020607

[9] Ochsner, N.K. and Gross, J.J. (2005) The Cognitive Control of Emotion. Trends in Cognitive Science, 9, $242-249$. http://dx.doi.org/10.1016/j.tics.2005.03.010

[10] Hot, P., Klein-Koerkamp, Y., Borg, Y., Richard-Mornas, A., Zsoldos, J., Paignon, A., Thomas Anterion, C. and Bacin, M. (2013) Fear Recognition Impairment in Early Stage Alzheimer's Disease: When Focusing on the Eyes Region Improves Performance. Brain and Cognition, 82, 25-34. http://dx.doi.org/10.1016/j.bandc.2013.02.001

[11] Guaita, A., Malnati, M., Vaccaro, R., Pezzati, R., Marcionetti, J., Vitali, S.F. and Colombo, M. (2009) Impaired Facial Emotion Recognition and Preserved Reactivity to Facial Expression in People with Severe Dementia. Archives of Gerontology and Geriatrics, 49, 135-146. http://dx.doi.org/10.1016/j.archger.2009.09.023

[12] Maki, Y., Yoshida, H., Yamaguchi, T. and Yamaguchi, H. (2013) Relative Preservation of the Recognition of Positive Facial Expression "Happiness" in Alzheimer's Disease. International Psychogeriatrics, 25, 105-110. http://dx.doi.org/10.1017/S1041610212001482

[13] Werheid, K., Gruno, M., Kathmann, N., Fischer, H., Almkvist, O. and Winblad, B. (2010) Biased Recognition of Positive Faces in Aging and Amnestic Mild Cognitive Impairment. Psychology and Aging, 25, 1-15. http://dx.doi.org/10.1037/a0018358

[14] Drapeau, J., Gosselin, N., Gagnon, L., Peretz, I. and Lorrain, D. (2009) Emotional Recognition from Face, Voice and Music in Dementia of the Alzheimer's Type: Implications for Music Therapy. The Neurosciences and Music III Disorders and Plasticity, Annals of the New York Academy of Sciences, 1169, 342-345. http://dx.doi.org/10.1111/j.1749-6632.2009.04768.x

[15] Moraitou, D., Papantoniou, G., Gkinopoulos, T. and Nigritinou, M. (2013) Older Adults' Decoding of Emotions: AgeRelated Differences in Interpreting Dynamic Emotional Displays and the Well-Preserved Ability to Recognize Happiness. Psychogeriatrics, 13, 139-147. http://dx.doi.org/10.1111/psyg.12016

[16] Johnson, R.D. and Whiting, L.W. (2012) Detecting Subtle Expressions: Older Adults Demonstrate Automatic and Controlled Positive Response Bias in Emotional Perception. Psychology and Aging, 28, 172-178. http://dx.doi.org/10.1037/a0029914

[17] Knight, M., Seymour, L.T., Gaunt, T.J., Baker, C., Nesmith, K. and Mather, M. (2007) Aging and Goal-Directed Emotional Attention: Distraction Reverses Emotional Biases. Emotion, 7, 705-714. http://dx.doi.org/10.1037/1528-3542.7.4.705

[18] Tomaszczyk, J., Fernandes, M.A. and MacLeod, C. (2008) Personal Relevance Modulates the Positivity Bias in Recall of Emotional Pictures in Older Adults. Psychonomic Bulletin \& Review, 15, 191-196. http://dx.doi.org/10.3758/PBR.15.1.191

[19] Kellough, J.L. and Knight, B.G. (2012) Positivity Effects in Older Adults' Perception of Facial Emotion: The Role of 
Future Time Perspective. The Journals of Gerontology, Series B: Psychological Sciences and Social Sciences, 67, 150158. http://dx.doi.org/10.1093/geronb/gbr079

[20] Moraitou, D. and Papantoniou, G. (2012) Mindfulness and Action Control in Young, Middle-Aged, and Old Adults: An Examination of Their Relationship to Attentional Bias towards Emotional Information. Research in Psychology (Ricerche di Psicologia), 2010, 591-626.

[21] Moraitou, D. and Efklides, A. (2007) Affect and Emotions. In: Fernandez-Ballesteros, R., Ed., GeroPsychology: European Perspectives for an Ageing World, Hogrefe \& Huber, Goettingen, 82-102.

[22] Henry, J.D., Ruffman, T., McDonald, S., Peek O’Leary, M., Phillips, L.H., Brodaty, H. and Rendell, P.G. (2008) Recognition of Disgust Is Selectively Preserved in Alzheimer's Disease. Neuropsychologia, 46, 1363-1370. http://dx.doi.org/10.1016/j.neuropsychologia.2007.12.012

[23] Ruffman, T., Henry, J.D., Livingstone, V. and Phillips, L.H. (2008) A Meta-Analytic Review of Emotion Recognition and Aging: Implications for Neuropsychological Models of Aging. Neuroscience \& Biobehavioral Reviews, 32, 863881. http://dx.doi.org/10.1016/j.neubiorev.2008.01.001

[24] Isaacowitz, D.M. and Stanley, J.T. (2011) Bringing an Ecological Perspective to the Study of Aging and Recognition of Emotional Facial Expressions: Past, Current, and Future Methods. Journal of Nonverbal Behavior, 35, 261-278. http://dx.doi.org/10.1007/s10919-011-0113-6

[25] Wright, C., Negreira, A., Gold, A., Britton, J., Williams, D. and Feldman Barrett, L. (2008) Neural Correlates of Novelty and Face-Age Effects in Young and Elderly Adults. NeuroImage, 42, 956-968. http://dx.doi.org/10.1016/j.neuroimage.2008.05.015

[26] Bar-Anan, Y., Wilson, T. and Gilbert, D. (2009) The Feeling of Uncertainty Intensifies Affective Reactions. Emotion, 9, 123-127. http://dx.doi.org/10.1037/a0014607

[27] Weierich, M., Wright, C., Negreira, A., Dickerson, B. and Feldman Barrett, L. (2010) Novelty as a Dimension in the Affective Brain. NeuroImage, 49, 2871-2878. http://dx.doi.org/10.1016/j.neuroimage.2009.09.047

[28] Carstensen, L., Fung, H. and Charles, S.T. (2003) Socioemotional Selectivity Theory and the Regulation of Emotion in the Second Half of Life. Motivation and Emotion, 27, 103-123. http://dx.doi.org/10.1023/A:1024569803230

[29] Charles, S.T. (2010) Strength and Vulnerability Integration: A Model of Emotional Well-Being acoss Adulthood. Psychological Bulletin, 136, 1068-1091. http://dx.doi.org/10.1037/a0021232

[30] Wyble, B., Sharma, D. and Bowman, H. (2008) Strategic Regulation of Cognitive Control by Emotional Salience: A Neural Network Model. Cognition and Emotion, 22, 1019-1051. http://dx.doi.org/10.1080/02699930701597627

[31] Yang, L. and Hasher, L. (2011) Age Differences in the Automatic Accessibility of Emotional Words from Semantic Memory. Cognition and Emotion, 25, 3-9. http://dx.doi.org/10.1080/02699930903523348

[32] Leclerc, C.M. and Kensinger, E.A. (2008) Effects of Age on Detection of Emotional Information. Psychology and Aging, 23, 209-215. http://dx.doi.org/10.1037/0882-7974.23.1.209

[33] Samanez-Larkin, G., Robertson, E., Mikels, J., Carstensen, L. and Gotlib, I. (2009) Selective Attention to Emotion in the Aging Brain. Psychology and Aging, 24, 519-529. http://dx.doi.org/10.1037/a0016952

[34] Scheibe, S. and Carstensen, L. (2010) Emotional Aging: Recent Findings and Future Trends. Journal of Gerontology Series B: Psychological Sciences and Social Sciences, 65B, 135-144. http://dx.doi.org/10.1093/geronb/gbp132

[35] Kelley, N., Tang, D. and Schmeichel, B.J. (2013) Mortality Salience Biases Attention to Positive versus Negative Images among Individuals Higher in Trait Self-Control. Cognition and Emotion, 28, 550-559. http://dx.doi.org/10.1080/02699931.2013.840269

[36] Isaacowitz, D., Waldinger, H., Goren, D. and Wilson, H. (2006) Selective Preference in Visual Fixation away from Negative Images in Old Age? An Eye-Tracking Study. Psychology and Aging, 21, 40-48. http://dx.doi.org/10.1037/0882-7974.21.1.40

[37] Kryla-Lighthall, N. and Mather, M. (2009) The Role of Cognitive Control in Older Adults' Emotional Well-Being. In: Bengston, V., Gans, D., Putney, N. and Silverstein, M., Eds., Handbook of Theories of Aging, Springer, New York, 323-344.

[38] Mather, M. and Knight, M. (2005) Goal-Directed Memory: The Role of Cognitive Control in Older Adults' Emotional Memory. Psychology and Aging, 20, 554-570. http://dx.doi.org/10.1037/0882-7974.20.4.554

[39] Ochsner, K.N. and Gross, J.J. (2007) The Neural Architecture of Emotion Regulation. In: Gross, J.J., Ed., Handbook of Emotion Regulation, Guilford, New York, 87-109.

[40] Ochsner, K.N., Ray, R.D., Cooper, J.C., Robertson, E.R., Chopra, S., Gabrieli, J.D. and Gross, J.J. (2004) For Better or for Worse: Neural Systems Supporting the Cognitive Down- and Up-Regulation of Negative Emotion. NeuroImage, 23, 483-499. http://dx.doi.org/10.1016/j.neuroimage.2004.06.030

[41] Folstein, M.F., Folstein, S.E. and McHugh, P.R. (1975) Mini-Mental State: A Practical Method for Grading the Cogni- 
tive State of Patients for the Clinician. Journal of Psychiatric Research, 12, 189-198. http://dx.doi.org/10.1016/0022-3956(75)90026-6

[42] Fountoulakis, K., Tsolaki, M., Chantzi, H. and Kazis, A. (2000) Mini-Mental State Examination (MMSE): A Validation Study in Greece. American Journal of Alzheimer's Disease and Other Dementias, 15, 342-345. http://dx.doi.org/10.1177/153331750001500604

[43] Sheikh, J.I. and Yesavage, J.A. (1986) Geriatric Depression Scale (GDS): Recent Evidence and Development of a Shorter Version. Clinical Gerontology, 5, 165-173.

[44] Fountoulakis, K., Tsolaki, M., Iacovides, A., Yesavage, J., O’Hara, R., Kazis, A. and Ierodiakonou, C. (1999) The Validation of the Short Form of the Geriatric Depression Scale (GDS) in Greece. Aging Clinical and Experimental Research (Milano), 11, 367-372. http://dx.doi.org/10.1007/BF03339814

[45] McDonald, S., Bornhofen, C., Shum, D., Long, E., Saunders, C. and Neulinger, K. (2006) Reliability and Validity of the Awareness of Social Inference Test (TASIT): A Clinical Test of Social Perception. Disability and Rehabilitation, 28, 1529-1542. http://dx.doi.org/10.1080/09638280600646185

[46] McDonald, S., Flanagan, S., Rollins, J. and Kinch, J. (2003) TASIT: A New Clinical Tool for Assessing Social Perception after Traumatic Brain Injury. Journal of Head Trauma Rehabilitation, 18, 219-238. http://dx.doi.org/10.1097/00001199-200305000-00001

[47] McDonald, S., Flanagan, S. and Rollins, J., the University of New South Wales' Media \& Education Technology Support Unit (2002) The Awareness of Social Inference Test (TASIT): Manual. Pearson, London.

[48] Nashiro, K., Sakaki, M. and Mather, M. (2012) Age Differences in Brain Activity during Emotion Processing: Reflections of Age-Related Decline or Increased Emotion Regulation? Gerontology, 58, 156-163. http://dx.doi.org/10.1159/000328465

[49] Brassen, S., Gamer, M. and Buechel, C. (2011) Anterior Cingulate Activation Is Related to a Positivity Bias and Emotional Stability in Successful Aging. Biological Psychiatry, 70, 131-137. http://dx.doi.org/10.1016/j.biopsych.2010.10.013

[50] Phillips, L., Scott, C., Henry, J.D., Mowat, D. and Bell, J.S. (2010) Emotion Perception in Alzheimer's Disease and Mood Disorder in Old Age. Psychology and Aging, 25, 38-47. http://dx.doi.org/10.1037/a0017369

[51] Goodkind, M.S., Sollberger, M., Gyurak, A., Rosen, H.J., Rankin, K.P., Miller, B. and Levenson, R. (2011) Tracking Emotional Valence: The Role of the Orbitofrontal Cortex. Human Brain Mapping, 33, 753-762. http://dx.doi.org/10.1002/hbm.21251

[52] Buhle, J.T., Silvers, J.A., Wager, T.D., Lopez, R., Onyemekwu, C., Kober, H., Weber, J. and Ochsner, K.N. (2013) Cognitive Reappraisal of Emotion: A Meta-Analysis of Human Neuroimaging Studies. Cerebral Cortex, Published Online. http://dx.doi.org/10.1093/cercor/bht154

[53] Freedman, M., Binns, M.A., Black, S.E., Murphy, C. and Stuss, D.T. (2013) Theory of Mind and Recognition of Facial Emotion in Dementia: Challenge to Current Concepts. Alzheimer Disease and Associated Disorders, 27, 56-61. http://dx.doi.org/10.1097/WAD.0b013e31824ea5db 
Scientific Research Publishing (SCIRP) is one of the largest Open Access journal publishers. It is currently publishing more than 200 open access, online, peer-reviewed journals covering a wide range of academic disciplines. SCIRP serves the worldwide academic communities and contributes to the progress and application of science with its publication.

Other selected journals from SCIRP are listed as below. Submit your manuscript to us via either submit@scirp.org or Online Submission Portal.
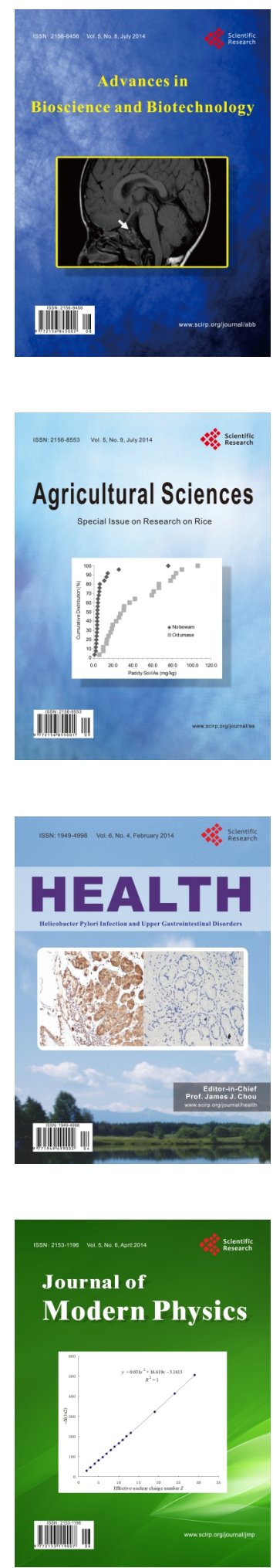
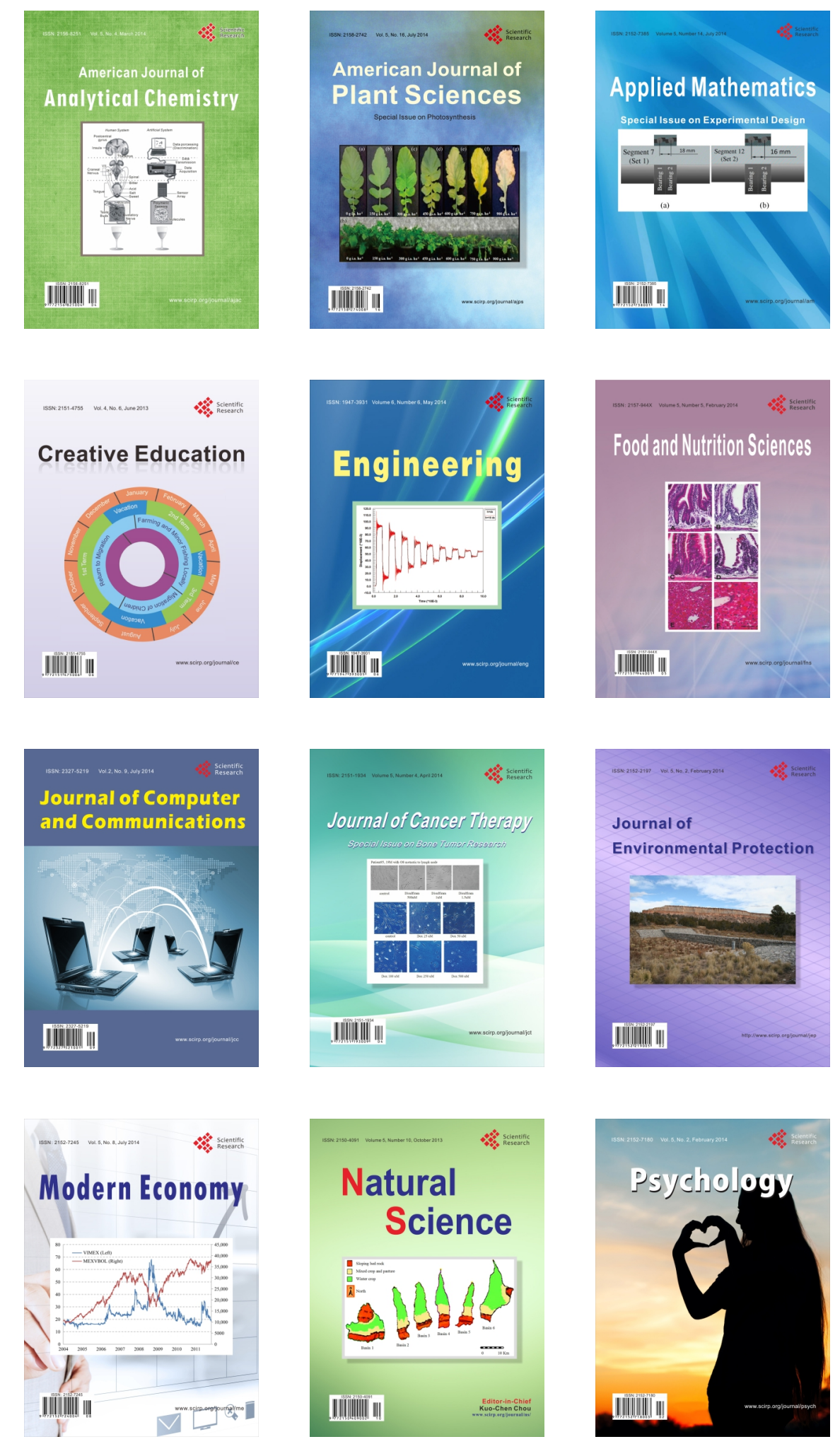